Ann. Génét. Sél. anim., I975, 7 (3), 33I-334.

NOTE

\title{
ENREGISTREMENT SUR UNE COURTE PÉRIODE DE LA CONSOMMATION D'ALIMENT CHEZ LA POULE PONDEUSE POUR L'ÉTUDE GÉNÉTIQUE DE L'EFFICACITÉ ALIMENTAIRE (')
}

\author{
A. BORDAS et P. MÉRAT \\ Laboratoire de Génétique factorielle, \\ Centre national de Recherches zootechniques, I. N.R. A., \\ 78350 Jouy en Josas (France)

\section{RÉSUMÉ}

Des corrélations ont été obtenues sur plusieurs lots expérimentaux, entre consommation alimentaire des pondeuses enregistrée sur une "longue " période ( 3 mois) et sur une période plus courte (I 6 jours), après correction pour le poids corporel et la production d'œufs durant l'ensemble des périodes. L'examen de ces corrélations suggère que la consommation " corrigée " estimée sur une durée d'environ 2 semaines peut être un critère de sélection suffisant sur l'efficacité de la conversion alimentaire.

L'efficacité de la conversion de l'aliment par les poules pondeuses dépend en grande partie de leur production d'œufs et de leur poids : aussi a-t-elle été améliorée indirectement par la sélection sur ces derniers critères. Cependant, il peut subsister, pour un même poids corporel et une même ponte, des différences dans l'ingestion alimentaire et dans l'utilisation métabolique des aliments ingérés. NORDSKog et al. (1972) ont passé en revue les observations dans ce domaine. Nous avons nous-mêmes (Bordas et MÉrAT, 1974) montré l'existence, dans plusieurs populations expérimentales, d'une variabilité génétique appréciable de la consommation alimentaire de pondeuses corrigée pour le poids et la production d'œufs pendant une période donnée. Ces résultats indiquent qu'une sélection directe sur ce critère pourrait donner un résultat, au moins dans certains troupeaux. Toutefois, l'enregistrement individuel de consommation pendant une durée importante et à l'échelle d'un élevage de sélection moderne est laborieux et coûteux. C'est pourquoi nous avons suggéré dans le même article quelques possibilités de sélection sur des critères

(1) Une partie de ces résultats a été présentée dans une Thèse d'Université soutenue par A. Bordas à la Faculté des Sciences, Paris VI. 
indirects simples, comme la taille d'appendices non emplumés ou la consommation d'eau. Cependant, ces derniers ne peuvent rendre compte que d'une fraction limitée de la variabilité du rendement alimentaire. Aussi avons-nous cherché, parallèlement, la possibilité d'utiliser des mesures de consommation individuelle sur de courtes périodes. La présente note expose nos résultats dans ce sens (cf. aussi Bordas, 1973).

Trois séries de lots expérimentaux ont été utilisées de 1970 à 1973 inclus :

- Poules de taille normale $\left(D w^{-}\right)$du troupeau expérimental élevé à Jouy en Josas (désigné par "Jouy").

- Poules naines $\left(d w^{-}\right)$du même troupeau.

- Souche " ponte" de type Rhode-Island (M99) originaire de la Station du Magneraud.

Les conditions d'expérimentation ont déjà été exposées dans notre précédent article (BORDAS et Mérat, 1974). Nous rappellerons seulement que l'âge des pondeuses en début de contrôle est de 8 mois environ et que l'aliment donné est de même formule d'une année à l'autre, à 16 p. Ioo de protéines et $2520 \mathrm{kcal} / \mathrm{kg}$ d'énergie métabolisable. Les lots "Jouy " considérés ici étaient éclos en automne et le contrôle de consommation avait lieu pour eux d'avril à fin juin de l'année suivante; les lots " M99 " naissaient au printemps et étaient contrôlés d'octobre à décembre.

Les poids corporels moyens allaient de 2090 à $2473 \mathrm{~g}$ suivant l'année pour les lots "Jouy $D w$ ", de I 588 à I 643 g pour les " Jouy dw", de I 827 à 2059 g pour les " M99 ". Les pourcentages moyens de ponte étaient compris entre 60 et $70 \mathrm{p}$. 100 pour les périodes considérées.

Pendant 3 périodes successives de 28 jours étaient enregistrés individuellement la consommation alimentaire, le poids corporel moyen, sa variation, le poids des œufs pondus. La moyenne de chaque variable sur l'ensemble des 3 périodes était prise en considération pour chaque individu. Nous la désignerons par $x$ (" mesure sur longue période ") en ce qui concerne la variable " consommation alimentaire ".

D'autre part, une durée de II jours consécutifs en I970, I6 les autres années, est envisagée séparément. Dans ce laps de temps (inclus à la fin de la dernière période de 28 jours) la consommation journalière d'aliment était notée, la quantité d'aliment dans chaque mangeoire étant pesée chaque jour à une heure aussi voisine que possible ( $9 \mathrm{~h}$ 30 du matin en moyenne) pour chaque poule. La consommation journalière moyenne de chaque individu pour l'ensemble de cette période sera désignée par $y$ (" mesure sur période courte ").

Les résultats obtenus portent sur deux points :

\section{I. - Corrélations entre mesure de la consommation alimentaire individuelle sur courte période $(y)$ et sur longue période $(x)$}

Outre l'indication des lots expérimentaux et des effectifs utilisés, le tableau I présente dans chaque cas la corrélation simple entre consommation mesurée sur courte et sur longue période. Tous les coefficients obtenus sont relativement élevés et hautement significatifs $(\mathrm{P}<\mathrm{o}, \mathrm{or})$.

La corrélation observée pour 1'année 1970 est significativement différente des autres, ce qui peut s'expliquer par le fait que la période "courte " de contrôle considérée pour cette année était de I I jours au lieu de 16 les autres années. L'ensemble des autres coefficients de corrélation, par contre, ne comporte pas d'hétérogénéité significative $\left(\chi^{2}=\mathrm{II}, \mathrm{I}\right.$ pour $6 \mathrm{~d}$.1., $\left.\mathrm{P} \simeq 0, \mathrm{IO}\right)$. Leur valeur moyenne, estimée pour l'ensemble des données à l'exclusion de 1970, est + o,84.

Le fait que la période restreinte de $I 6$ jours soit incluse dans la période totale considérée entraîne nécessairement une corrélation positive entre les deux (dans la mesure du moins où il n'y a pas de corrélation négative appréciable entre la consommation individuelle pendant la période de 16 jours et la période antérieure) ce qui n'est pas un inconvénient dans la mesure où c'est la consommation " totale " dont l'estimation nous intéresse. Cette corrélation " automatique " entre partie et tout peut être estimée à une valeur inférieure à 0,2 ; elle ne rend donc compte que très partiellement de la corrélation observée. 
D'autre part, cette dernière concerne les consommations non corrigées pour le poids corporel et la masse d'œufs produite, de sorte qu'elle peut refléter en partie la corrélation existant, pour chacun de ces deux caractères, entre sa mesure à deux périodes successives pour le même individu (nous ne prenons pas en considération la variation de poids, qui avait peu d'incidence en " période courte ») (1). Pour éliminer ce facteur parasite (du point de vue de l'estimation de la consommation " corrigée "), nous présentons, dans la dernière colonne du tableau $r$, la corrélation partielle entre les deux consommations définies plus haut, en fixant les deux variables " poids corporel moyen " (sur l'ensemble des périodes) et " poids d'œufs pondus " (également sur l'ensemble des périodes). Les coefficients de corrélation partielle des divers lots ne présentent pas d'hétérogénéité significative dans l'ensemble, y compris celui obtenu en $1970\left(\chi^{2}=8,4\right.$ pour 7 d.1. $)$. La valeur $+0,70$ obtenue au total, quoiqu'un peu inférieure à la corrélation simple, reste assez élevée.

\section{TABLEAU I}

Corrélations entre consommation alimentaire des pondeuses sur une courte période $(y)$ et sur une longue période $(x)$

\begin{tabular}{|c|c|c|c|}
\hline \multirow[b]{2}{*}{ Année et population } & \multirow[b]{2}{*}{$N$} & \multicolumn{2}{|c|}{ Coefficient de corrélation } \\
\hline & & $\begin{array}{c}\text { simple } \\
\left(r_{x y}\right)\end{array}$ & $\begin{array}{c}\text { partiel } \\
\text { (à poids corporel } \\
\text { et production } \\
\text { d'œufs fixés) }\end{array}$ \\
\hline \multicolumn{4}{|l|}{ "Jouy " $(D w)$} \\
\hline $1970(1) \ldots \ldots$ & 63 & $+0,66$ & $+0,76$ \\
\hline $1971 \ldots \ldots \ldots$ & 76 & $+0,82$ & $+0,72$ \\
\hline $1972 \ldots \ldots \ldots$ & 51 & $+0,92$ & $+0,82$ \\
\hline $1973 \ldots \ldots \ldots$ & 29 & $+0,82$ & $+0,68$ \\
\hline \multicolumn{4}{|l|}{ "Jouy " (dw) } \\
\hline $1972 \ldots \ldots \ldots$ & 52 & $+0,88$ & $+0,75$ \\
\hline $1973 \ldots \ldots \ldots$ & 29 & $+0,87$ & $+0,46$ \\
\hline \multicolumn{4}{|l|}{ M 99} \\
\hline $1971 \ldots \ldots \ldots$ & 94 & $+0,81$ & $+0,71$ \\
\hline $1972 \ldots \ldots$ & 65 & $+0,77$ & $+0,65$ \\
\hline \multicolumn{4}{|l|}{ Total } \\
\hline 1970 inclus $\ldots \ldots$ & & $+0,81$ & $+0,70$ \\
\hline - non inclus .. & & $+0,84$ & $+0,71$ \\
\hline
\end{tabular}

(1) Période "courte" de 11 jours seulement (16 jours les autres années).

\section{2. - Répétabilité de la consommation alimentaive journalière}

En complément de ces résultats, nous avons les mêmes années estimé la répétabilité de la consommation alimentaire journalière enregistrée sur les périodes " courtes " de II ou I6 jours. Sur l'ensemble des données, la répétabilité est égale à o,49. Celle de la consommation d'eau, estimée

(i) Il faut noter que cette variation de poids n'était pas corrigée pour la présence ou non d'un œuf dans l'utérus au moment de la pesée. 
en même temps, est pour le total des lots égale à o,69. La valeur plus élevée obtenue pour la consommation d'eau se retrouve chaque année.

En conclusion, pour sélectionner, à ponte et à poids corporel déterminés, les poules les plus " économes " d'aliment, il apparaît donc qu'une appréciation de la consommation de chacune sur une quinzaine de jours serait une assez bonne indication. Cette consommation devrait, connaissant le poids corporel et la production d'œufs pendant la même période, être " corrigée "à l'aide d'une équation de régression du type de celle proposée par BYERLY (I94I).

Reçu pour publication en août 1975.

\title{
REMERCIEMEN'TS
}

Nous remercions le Dr J. Guillaume, Station de Recherches avicoles, I. N. R. A., 37 Nouzilly, de ses utiles suggestions concernant ce manuscrit.

\section{SUMMARY}

\author{
SHORT-PERIOD MEASUREMENT OF FEED INTAKE \\ IN THE LAYING HEN FOR A GENETIC STUDY OF FEED EFFICIENCY
}

Correlations were obtained within several experimental groups, between the feed Consumption of laying hens measured over a "long " period ( 3 months) and that measured over a shorter period (I 6 days), after correction for body weight and egg mass produced during the whole period. The observed values for these correlations suggest that " corrected " feed consumption estimated over about 2 weeks can be a sufficient selection criterion for feed conversion efficiency.

\section{RÉFÉRENCES BIBLIOGRAPHIQUES}

Bordas A., 1973. Influence du génotype sur la consommation alimentaire chez les poules pondeuses. Thèse, Univ. Paris VI.

Bordas A., MÉnat P., I974. Variabilité génétique et corrélations phénotypiques caractérisant la consommation alimentaire de poules pondeuses après correction pour le poids corporel et la ponte. Ann. Génét. Sél. anim., 6, 369-379.

BYERLY T. C., r94r. Feeds and other costs of producing market eggs. Bull., no Ar, Univ. Maryland Agric. Exp. Sta.

Nordskog A. W., French H. L. jr, Arboleda C. R., Casey D. W., i972. Breeding for efficiency of egg production. World Poult. Sci. J., 29, I75-188. 